\title{
Forced expression of Nanog with mRNA synthesized in vitro to evaluate the malignancy of HeLa cells through acquiring cancer stem cell phenotypes
}

\author{
YAN DING, AI QING YU, XIAO LI WANG, XING RONG GUO, YA HONG YUAN and DONG SHENG LI \\ Hubei Key Laboratory of Embryonic Stem Cell Research, Taihe Hospital, \\ Hubei University of Medicine, Shiyan, Hubei 442000, P.R. China
}

Received July 29, 2015; Accepted October 5, 2015

DOI: $10.3892 /$ or.2016.4639

\begin{abstract}
Nanog is a pluripotency-related factor. It was also found to play an important role in tumorigenesis. To date, the mechanisms underlying cervical tumorigenesis still need to be elucidated. In the present study, Nanog mRNA was synthesized in vitro and transfected into HeLa cells. After mRNA transfection, the forced expressed of Nanog in HeLa cells led to markedly increased invasion, migration, resistance to chemotherapeutic agents and dedifferentiation. In a subcutaneous xenograft assay, these cells had significantly increased tumorigenic capacity. Real-time PCR indicated that Nanog-induced dedifferentiation was associated with increased expression of endogenous Oct4, Sox 2 and FoxD3. In addition, the dedifferentiated HeLa cells acquired features associated with cancer stem cells (CSCs), such as multipotent differentiation capacity, and expression of CSC markers such as CD133. These data imply that Nanog is a positive regulator of cervical cancer dedifferentiation.
\end{abstract}

\section{Introduction}

Cervical cancer is one of the most common female cancers and is associated with a high mortality rate (1). In the last few years, there has been great progress in the early diagnosis of cervical cancer along with the improvement in biological detection technology. The universal detection of cervical cancer and high-risk HPV for women of childbearing age have greatly improved the rate of early diagnosis, and the treatment efficiency of early stage cervical cancer is very good. However, the treatment efficiency of advanced cervical cancer is still very poor, and the 5-year survival rate is also very low.

Correspondence to: Dr Dong-Sheng Li, Hubei Key Laboratory of Embryonic Stem Cell Research, Taihe Hospital, Hubei University of Medicine, Shiyan, Hubei 442000, P.R. China

E-mail: dsli1698@126.com

Key words: Nanog, cervical cancer, mRNA
Therefore, it is necessary to further explore the pathogenesis of cervical cancer.

Cancer stem cells (CSCs) appear to be responsible for tumor initiation, progression and resistance to conventional treatment $(2,3)$. Nanog is one of the core transcription factors for maintaining the stemness of embryonic stem cells (ESCs) (4). Abnormal expression of Nanog is associated with several types of cancers, such as glioma (5), breast cancer (6), embryonic carcinoma (7), and prostate cancer (8). Jeter et al (9) reported that cytoplasmic $\mathrm{Nanog}^{+}$stromal cells could promote cervical cancer progression. Our previous study also indicated that a TALEN-mediated Nanog gene knockout could attenuate the malignancy of HeLa cells (10). These results suggested that Nanog is a risk factor for cervical cancer. However, whether Nanog is associated with cervical cancer cell dedifferentiation and how cervical cancer cells acquire the ability to invade surrounding tissues and metastasize are still unclear.

In the present study, to elucidate the role of Nanog in cervical cancer progression, we used mRNA synthesized in vitro for the forced expression of Nanog. This synthetic mRNA could bypass innate anti-viral responses induced in human cell dedifferentiation and had kinetics substantially superior to established viral protocols (11-13). Furthermore, this method was non-mutagenic (2).

\section{Materials and methods}

Cell culture. HeLa cells (10) were maintained in Dulbecco's modified Eagle's medium (DMEM) containing 10\% FBS.

Nanog mRNA synthesized in vitro. In vitro transcription template construction and RNA synthesis is schematized in Fig. 1A according to introduction of Zangi et al (13). The ORF of Nanog was templated from the cDNA of HN4 human embryonic stem cells (hESCs) (14). PCR reactions were performed using PCR Master Mix (R300A; Takara) according to the manufacturer's instructions. Splint-mediated ligations were carried out using T4 DNA ligase (Oncogene). All intermediate PCR and ligation products were purified using QIAquick spin columns (Qiagen) before further processing. Template PCR amplifications were sub-cloned using the pcDNA 3.3-TOPO TA cloning kit (Invitrogen). Plasmid inserts were excised 
by restriction digestion and recovered with Size Select gels (Invitrogen) and then used to template tail PCRs.

RNA was synthesized with the MEGAscript T7 kit (Ambion), using $1.6 \mu \mathrm{g}$ of purified tail PCR product to template each $40 \mu 1$ reaction. Ribonucleoside blend, composed of 3'-0-Me-m7G (5') ppp (5') G ARCA cap analog (New England Biolabs), adenosine triphosphate and guanosine triphosphate (USB, Cleveland, OH, USA), 5-methylcytidine triphosphate and pseudouridine triphosphate (TriLink Biotechnologies, San Diego, CA, USA), were used. Reactions were incubated for $5 \mathrm{~h}$ at $37^{\circ} \mathrm{C}$ and then treated with antarctic phosphatase (New England Biolabs) for $2 \mathrm{~h}$ at $37^{\circ} \mathrm{C}$ to remove residual 5 '-triphosphates. Treated RNA was purified and quantitated using a Nanodrop (Thermo Scientific, Waltham, MA, USA).

Nanog RNA transfection and stability assay. RNA transfection was carried out using TransIT-mRNA (Mirus Bio, Madison, WI, USA) cationic lipid delivery vehicles. For TransIT mRNA transfections, $\sim 1 \mu \mathrm{g}$ mRNA was added to $100 \mu \mathrm{l}$ Opti-MEM and mixed gently. BOOST reagent was added $(1 \mu \mathrm{l} / \mathrm{microgram}$ of RNA) followed by TransIT-mRNA ( $1 \mu \mathrm{l} / \mathrm{microgram}$ of RNA), and the RNA-lipid complexes were added to the culture media after a 2-min incubation at room temperature (RT). Cells were cultured for an additional 36 to $48 \mathrm{~h}$ before repeating the same mRNA transduction procedure. Three additional mRNA transduction experiments were performed.

To determine the half-life of Nanog mRNA, total protein of HeLa cells was extracted at different time-points $(0,6$, 18,30 and $42 \mathrm{~h}$ ) after mRNA transfection. Nanog and $\beta$-actin gene expression levels at each time-point were measured by western blotting.

Tumor tissue species collection and immunohistochemistry. Cervical cancer specimens (ten for high- and low-grade group, respectively) were collected from patients who underwent cervicectomy for cervical cancer between 2010 and 2014 at Taihe Hospital, the Affiliated Hospital of Hubei University of Medicine. The histological type of cervical cancer was determined according to WHO classification criteria. Formalin-fixed, paraffin-embedded sections of tumors and adjacent non-tumorous tissues were used to detect the expression of Nanog. The present study was approved by the Ethics Committee of Taihe Hospital.

Cisplatin and paclitaxel treatment and cell viability assay. Cell viability was determined using an MTS kit (Promega). Freshly disassociated cells were seeded in 96-well plates (2x10 $/$ well; Corning), and the media were replaced with HeLa cell medium containing $1 \mu \mathrm{g} / \mathrm{ml}$ cisplatin or $20 \mathrm{ng} / \mathrm{ml} \mathrm{pacli-}$ taxel the next day. After treatment for 24 to $48 \mathrm{~h}, 20 \mu \mathrm{l}$ MTS working solution was added to the medium and incubated at RT for 1-4 h, and then the OD value at $490 \mathrm{~nm}$ was recorded. Five replicate wells were used for each group.

Cell invasion ability detection. Transwell filters were used to analyze cell migration in vitro (10). The upper chamber of the polycarbonate membrane filter inserts $(8-\mu \mathrm{m}$; Corning, USA) were pre-coated with Matrigel, and then a total of $5 \times 10^{4}$ cells was seeded into a 24-well plate and cultured in $200 \mu 1 \mathrm{DMEM}$ medium. The lower chamber was filled with $500 \mu \mathrm{l}$ of HeLa cell
A
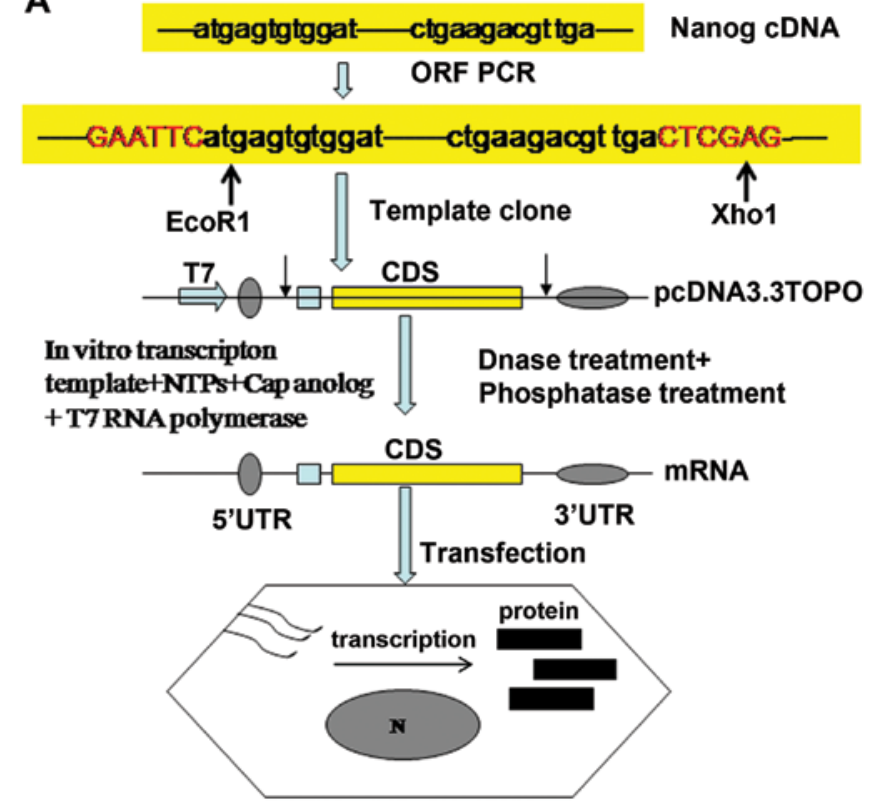

B

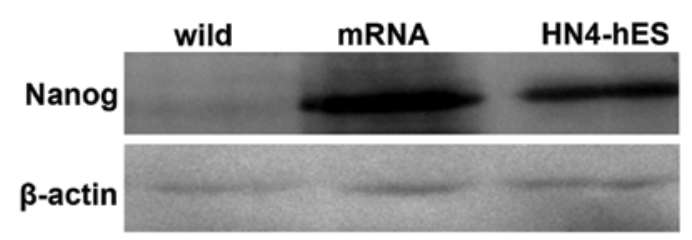

Figure 1. Disruption of Nanog mRNA synthesized in vitro. (A) Schematic representation of the Nanog mRNA synthesized in vitro and its modification. (B) Western blotting to test the activation of Nanog mRNA, with human embryonic stem cells (hESCs)-HN4 as the positive control.

culture medium. After incubation for $48 \mathrm{~h}$, non-migrated cells in the upper chamber surface were removed, and the migrated cells on the bottom side of the membrane were fixed and then stained with $0.1 \%$ Crystal violet. The stained membranes were observed, and the number of migrated cells was counted.

Migration ability detection. The migration ability of HeLa cells was tested as described in our previous study (10). A total of $5 \times 10^{5}$ cells was seeded into 6 -well plates, and a $10 \mu 1$ pipette tip was used to create a scratch on the cell monolayer the next day. Then, the cells were cultured with DMEM and incubated at $37^{\circ} \mathrm{C}$ in $5 \% \mathrm{CO}_{2}$ atmosphere for $48 \mathrm{~h}$. Pictures were taken at 0,24 and $48 \mathrm{~h}$. Distance from the scratch was measured from one side to the other. Three replicate wells were used for each group.

Tumor sphere forming ability detection. Cells were digested and resuspended in DMEM/F12 medium at a density of 1,000 cells $/ \mathrm{ml}$ and seeded into 6 -well plates $(2 \mathrm{ml} /$ well $)$. The serum-free culture medium consisted of DMEM/F12 (Sigma), $0.4 \% \mathrm{BSA}, 2 \% \mathrm{~B} 27$ (Invitrogen), $20 \mathrm{ng} / \mathrm{ml}$ human recombinant fibroblast growth factor 2 (FGF-2) and epidermal growth factor (EGF) (both from Sigma). After 10-14 days of culture, sphere number was measured. Each group was plated in 3 duplicate wells.

Colony-formation ability detection. The colony-formation assay was performed in 12 -well plates. Cells were seeded at 
A

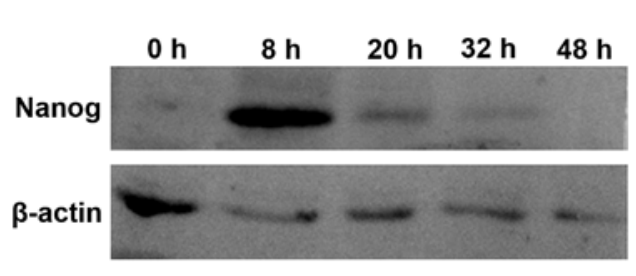

C
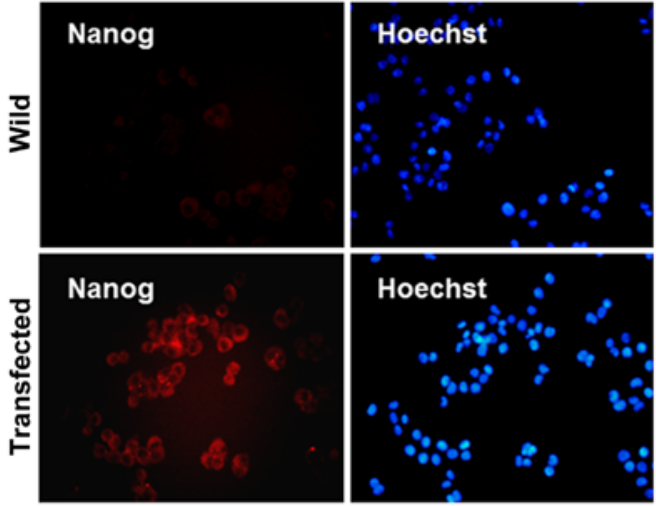

B
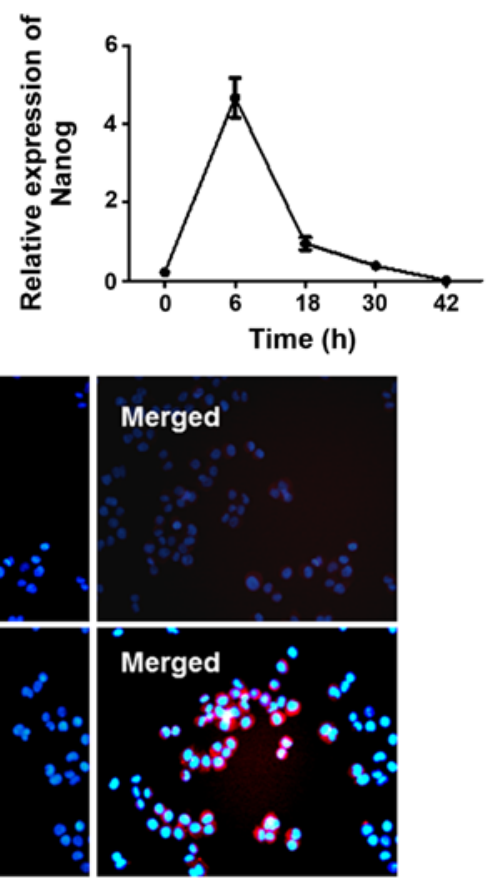

Figure 2. Activity of Nanog mRNA synthesized in vitro. (A) Western blotting to examine the half-life of Nanog mRNA. (B) The relative concentration of Nanog protein at different time-points. It was shown that the half-life of Nanog mRNA is $\sim 42 \mathrm{~h}$. (C) Immunofluorescence detection of Nanog expression before or $20 \mathrm{~h}$ after Nanog mRNA transfection.

1,000 cells/well and cultured with normal HeLa cell medium until colonies were large enough to be visualized. Then, colonies were fixed and stained with $0.1 \%$ crystal violet. Experiments were done in triplicate.

Subcutaneous xenograft. HeLa cells $\left(1 \times 10^{6}\right)$, transfected with or without Nanog mRNA, were injected subcutaneously into nude mice $(n=3)$ as described in our previous study (10) and observed weekly until the mice died.

Immunofluorescence. Cells were fixed with $4 \%$ paraformaldehyde followed by permeabilization with $0.1 \%$ Triton X-100 and then blocked in 5\% BSA (both from Sigma). Afterwards, the cells were incubated with primary Nanog (1:100) and CD133 (1:200) (both from Abcam) antibodies overnight at $4^{\circ} \mathrm{C}$ and then with FITC-conjugated with rat anti-mouse immunoglobulin (1:500) and Cy3-rat anti-mouse (1:500) secondary antibody for $30 \mathrm{~min}$ at RT. Finally, the nuclei were stained with $1 \mu \mathrm{g} / \mathrm{ml}$ Hoechst 33342 and visualized using a Leica microscope.

Real-time (RT)-PCR and western blot assay. Real-time (RT)-PCR, western blot assay and statistical analysis were performed as described in our previous study (10).

\section{Results}

Nanog mRNA activated the expression of endogenous Nanog. We transfected HeLa cells with Nanog mRNA, synthesized in vitro, and detected the expression of endogenous Nanog. First, we measured the protein level of Nanog in HeLa cells at $6,18,30$ and $42 \mathrm{~h}$ after transfection using western blotting and immunofluorescence. Nanog protein expression was evident $6 \mathrm{~h}$ after transfection and was maintained for up to $30 \mathrm{~h}$. Forty-two hours after transfection, Nanog levels returned to baseline (Figs. 1B and 2). HeLa cells were treated with Nanog mRNA 4 times every other $32 \mathrm{~h}$. Then the cells were cultured for an additional 8-10 days, the protein level of Nanog was detected, and it remained at a high level.

Nanog expression correlated with malignancy and prognosis of cervical cancer patients. Nanog expression in cervical cancer specimens and adjacent non-cancer tissues from 40 patients with cervical cancer (20 each for low- and high-grade specimens) was detected by immunohistochemistry. The expression of Nanog could hardly be detected in non-cancer tissue specimens. On the contrary, Nanog expression could be detected in $20 \%$ of low-grade and $45 \%$ of high-grade cervical cancer specimens. This indicated that the expression of Nanog was positively correlated with clinical grading and malignant degree of cervical cancer (Fig. 3).

Nanog induces more aggressive biological behavior. Transwell cell migration assays indicated that forced expression of Nanog significantly increased the invasive ability of HeLa cells. As shown in Fig. 3, after 48 h, the number of transfected cells that passed through the Transwell was $138 \pm 16$, but the number of passed cells in the wild-type group was $62 \pm 7 \quad(\mathrm{P}<0.05$, Fig. 4A and B), which was significantly lower than in the transfected group.

Scratch assays indicated that Nanog overexpression resulted in a significant increase in HeLa cell migration. The migration rates of transfected cells after 24 and $48 \mathrm{~h}$ were $26.8 \pm 3.4$ and $58.7 \pm 7.2 \%$, respectively. However, the migration 

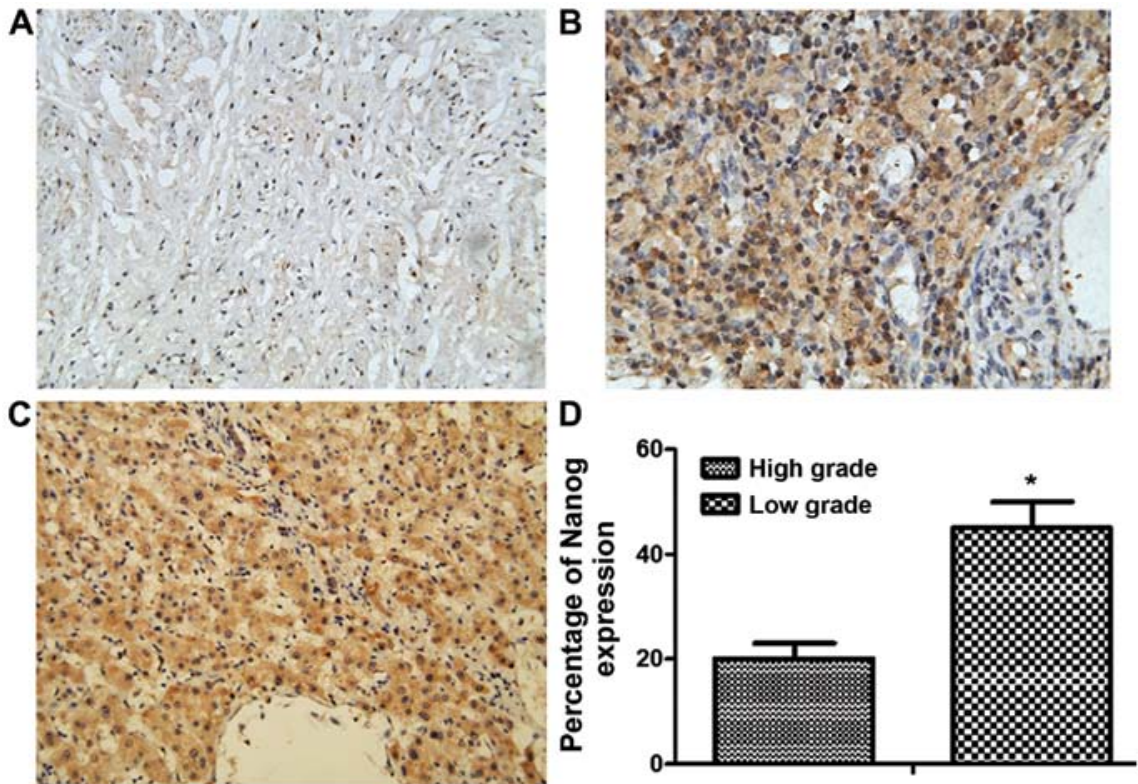

Figure 3. Correlation of Nanog expression and clinical grading of cervical cancer. (A-C) Immunohistochemistry detection of the expression of Nanog in different grades of cervical cancer tissues (ten for high- and low-grade group, respectively). (A) Normal control, (B) high-grade, (C) low-grade. (D) Percentage of Nanog expression in low-grade and high-grade cervical cancer. ${ }^{*} \mathrm{P}<0.05$.

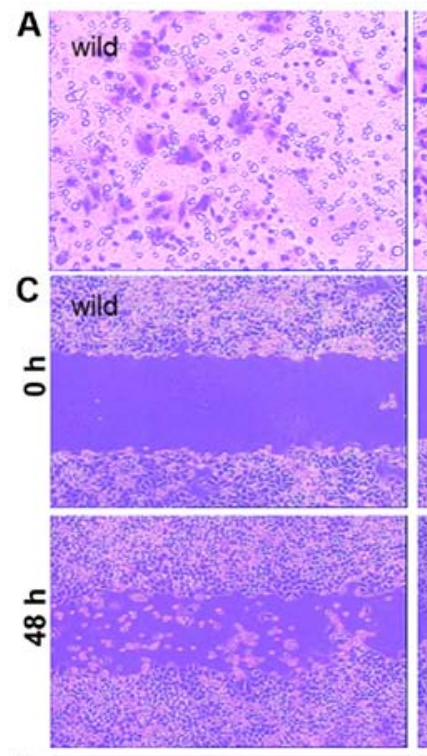

E

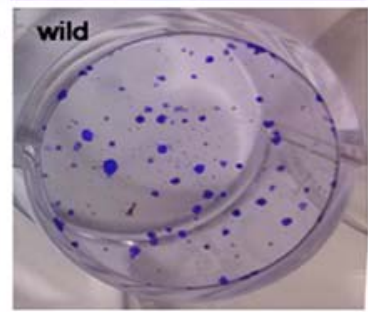

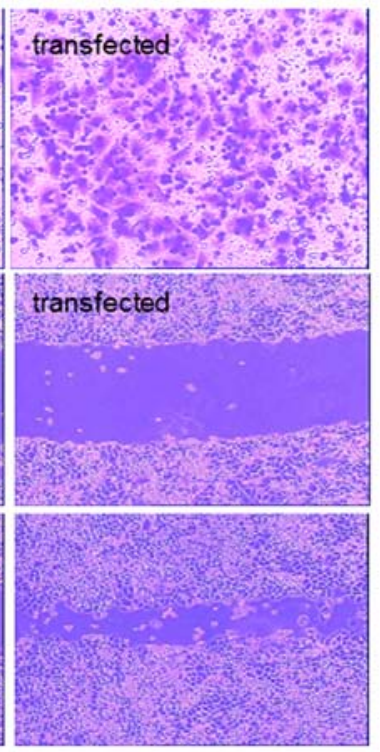

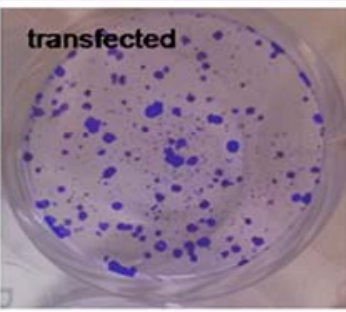

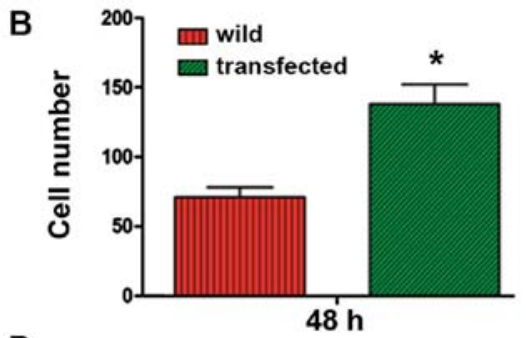

D

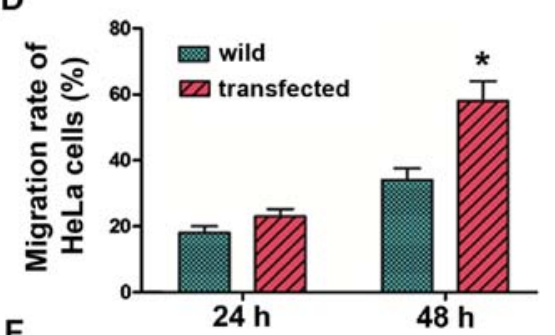

$\mathbf{F}$

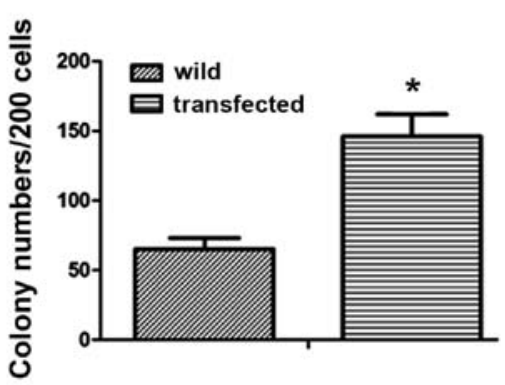

Figure 4. The effect of forced expression of Nanog on HeLa invasiveness. (A) Invasive cells were stained by crystal violet. (B) The number of invasive cells in the transfected group was $138 \pm 15$, which was significantly higher than the wild-type control cells ( $\mathrm{P}<0.05)$. (C) The scratch assay showed that the migration distance of transfected HeLa cells was greatly increased compared with the wild-type group. (D) The difference in migration distance of Nanog forced expression cells compared with the wild control cells at 24 and $48 \mathrm{~h}$. The difference in migration between Nanog overexpressing cells and the control was statistically significant at $48 \mathrm{~h}\left({ }^{*} \mathrm{P}<0.05\right)$. (E) Crystal violet staining analysis of the colony formation ability of wild and transfected cells. (F) The number of colonies formed by Nanog knockdown cells was lower than the control cells $\left({ }^{*} \mathrm{P}<0.05\right)$.

rates in wild-type group were $18.9 \pm 2.3$ and $33.2 \pm 4.1 \%$, respectively $(\mathrm{P}<0.05$, Fig. $4 \mathrm{C}$ and $\mathrm{D})$.
As shown in Fig. 4E and F, clonogenicity of transfected HeLa cells was increased according to the number of cell colo- 


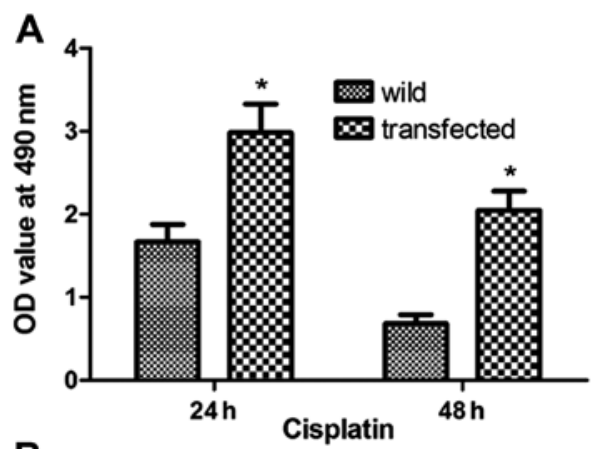

C
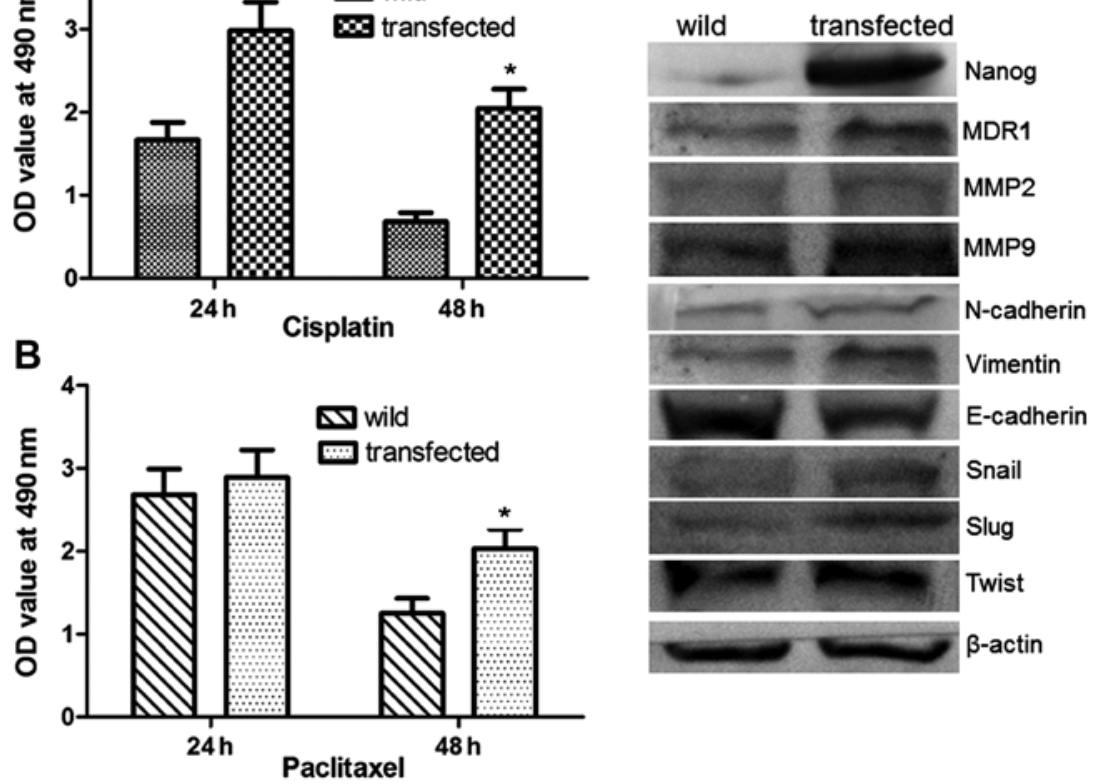

Figure 5. The effect of Nanog on the chemosensitivity and EMT ability of HeLa cells. (A and B) The wild and transfected HeLa cells were exposed to cisplatin (A, $1 \mu \mathrm{g} / \mathrm{ml})$ and paclitaxel (B, $40 \mathrm{ng} / \mathrm{ml})$ for 24 and $48 \mathrm{~h}$, and the viability was accessed by an MTS kit. Forced expression of Nanog resulted in lower chemosensitivity to cisplatin and paclitaxel compared with the control cells. "P<0.05. (C) Western blot analysis of the level of MDR1- and EMT-associated proteins in HeLa cells transfected with and without Nanog mRNA.
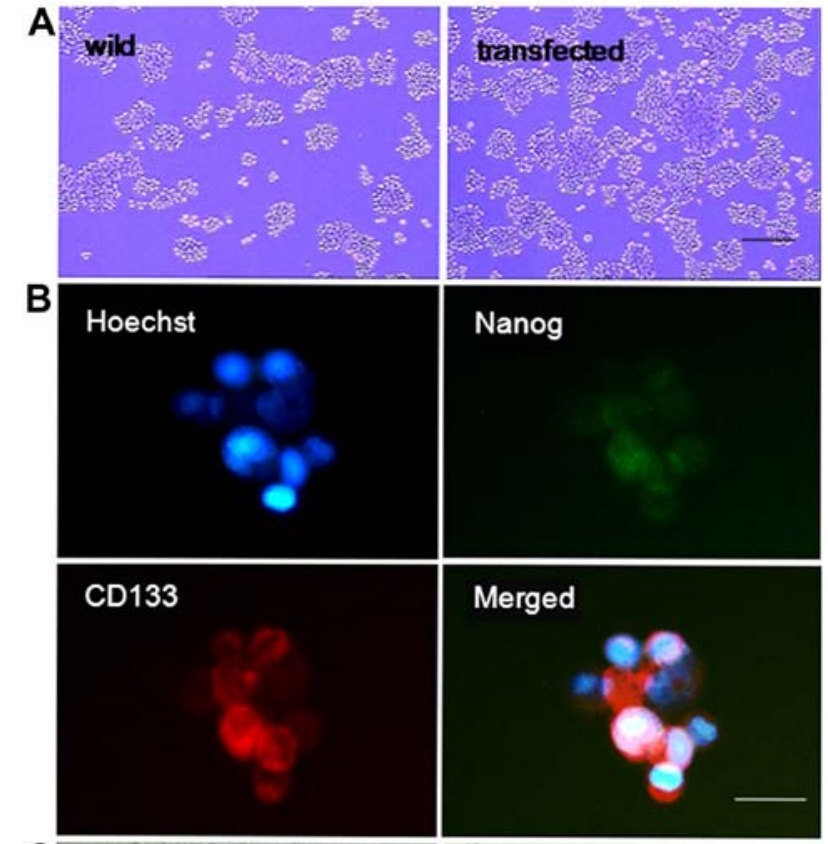

C
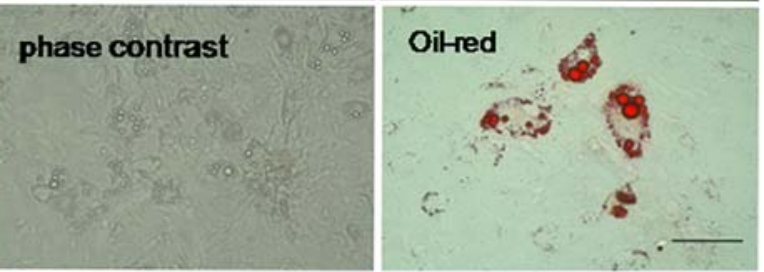

Figure 6. The effect of Nanog forced expression on acquiring CSC-like phenotype ability. (A) Wild and transfected HeLa cells were induced to form tumor spheres with serum-free medium for 10-12 days. (B) Immunofluorescence detected the expression of Nanog and stem cell marker, CD133, in tumor sphere cells. (C) The tumor sphere cells were further induced to lipid cells. After induction for 15 days, lipid droplets were confirmed with oil red-O staining. Scale bar, $200 \mu \mathrm{m}$. nies, and the colony number of transfected cells was $143 \pm 15$, higher than the wild-type group, which was $68 \pm 8(\mathrm{P}<0.05)$.

To evaluate the effect of Nanog on the sensitivity of HeLa cells to chemotherapy, the wild and transfected cells were exposed to cisplatin or paclitaxel. As shown in Fig. 5A and B, Nanog mRNA transfected HeLa cells were less sensitive to cisplatin and paclitaxel than the controls. These data indicated that the sensitivity of HeLa to chemotherapy drugs was decreased by forced expression of Nanog.

To further evaluate the mechanism by which Nanog affects the aggressive biological behavior and chemotherapy sensitivity of HeLa cells, we investigated the expression of MDR1, which is regarded as an important factor in drug resistance and sensitivity of chemotherapy, MMP2, MMP9, and EMT associated signals. As shown in Fig. 5C, compared with the wild-type HeLa cells, the expression of MDR1, MMP2, MMP9, N-cadherin, vimentin, Snail, Slug, and Twist were significantly increased, but the expression of E-cadherin was decreased in Nanog mRNA-transfected cells $(\mathrm{P}<0.05)$.

Nanog-induced dedifferentiation of cervical cancer cells and its mechanism. Cells transfected with and without Nanog mRNA were cultured in tumor sphere-forming medium. After 14 days, the number and size of the tumor spheres were analyzed. As shown in Fig. 6A, Nanog mRNA-transfected cells formed significantly more tumor spheres than control cells. Furthermore, the size of the tumor spheres was also significantly larger in cells with forced Nanog expression.

Immunofluorescence was used to detect the expression of Nanog and CD133 in sphere-forming cells, and it was found that $>90 \%$ of these cells expressed both markers (Fig. 6B). To further confirm their cancer stemness, the tumor sphere HeLa 

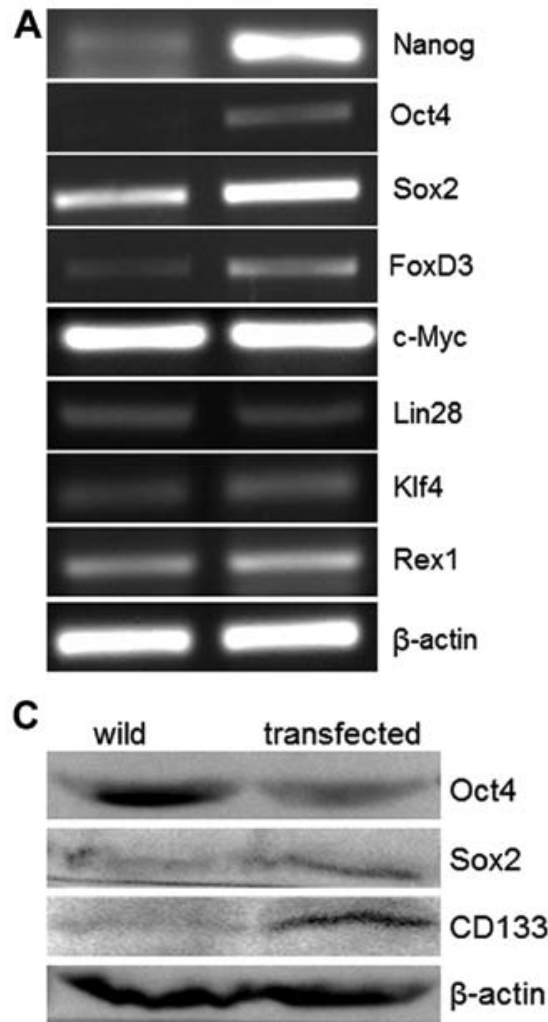

B

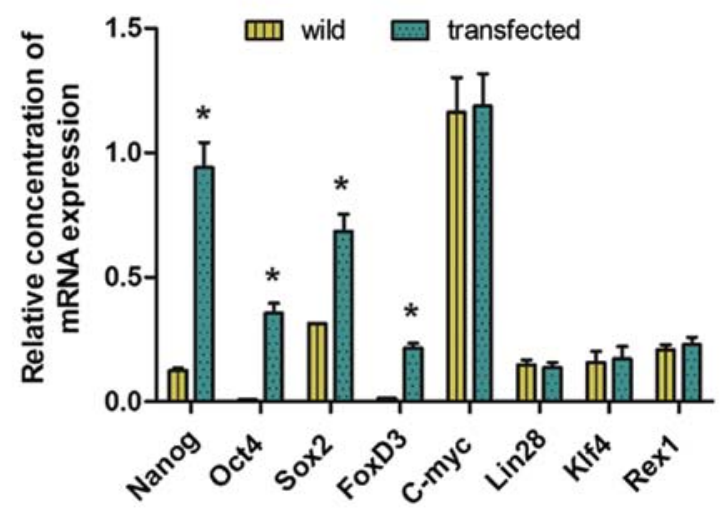

Figure 7. Mechanisms underlying Nanog-induced differentiation. (A and B) Quantitative RT-PCR was performed for Nanog, Oct4, Sox2, c-Myc, KLF4, Lin28, Rex1 and FoxD3 ( $\mathrm{n}=3$ ), and showed significant increase of Nanog, Oct4, Sox2 and FoxD3 ("P<0.05), but not Rex1, c-Myc, Klf4 and Lin28. (C) Western blot to detect the expression of Oct4, Sox 2 and CD133 in control and Nanog overexpressing HeLa cells.

A

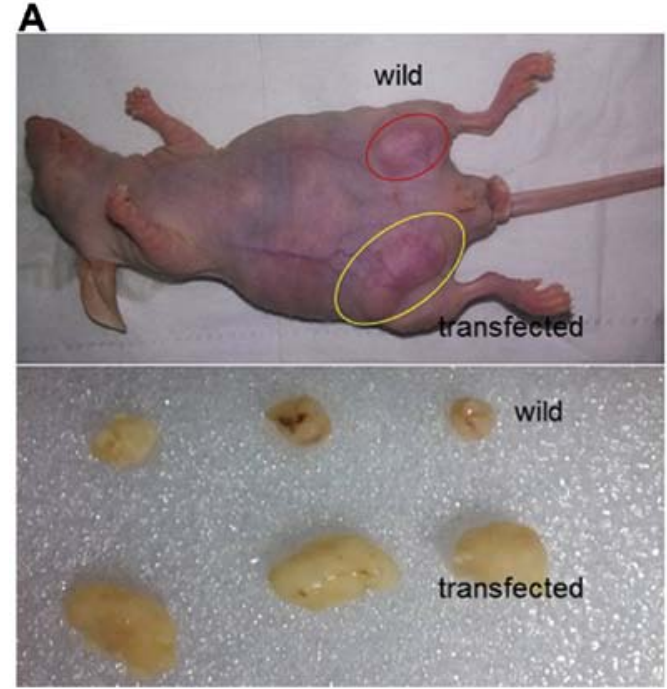

B

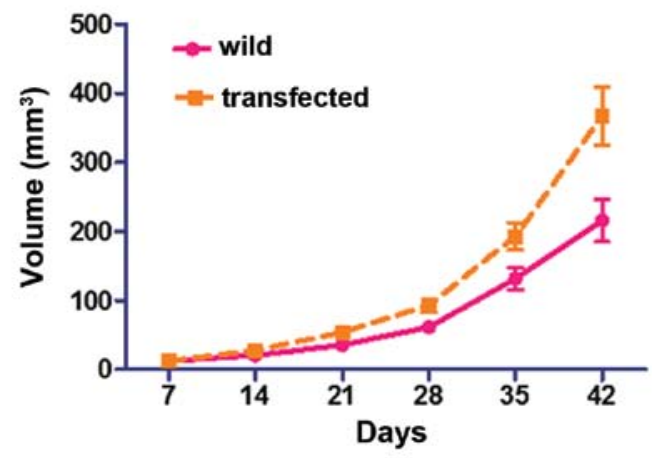

Figure 8. The effect of forced Nanog expression on tumorigenicity of HeLa cells. (A) Wild or transfected cells $\left(1 \times 10^{6}\right)$ were injected subcutaneously into the nude mice $(n=3)$, and these mice were followed. (B) The tumor volume of neoplasms in wild and transfected group from day 0 to 35 .

cells were cultured in adipogenic differentiation media for another 15 days and then stained with oil red-O. As shown in Fig. 6C, the red lipid droplets were observed in the differentiated cancer cells.

RT-PCR detected the expression of other transcription factors involved in somatic cell reprogramming. It was found that the expression levels of endogenous Oct4, Sox 2 and FoxD3 were significantly increased, but Rex1, Klf4, Lin28 and c-Myc were not increased (Fig. 7A and B). Western blotting also detected increased expression of Oct4, Sox 2 and CD133 in Nanog mRNA-transfected HeLa cells (Fig. 7C).

Increased tumorigenicity after Nanog-induced dedifferentiation. In order to verify the effect of Nanog-mediated dedifferentiation in vivo, nude mice $(\mathrm{n}=3)$ were transplanted with HeLa cells transfected with or without Nanog mRNA $\left(1 \times 10^{6}\right)$ subcutaneously and were then monitored for tumor growth. As shown in Fig. 8, the tumors formed by Nanog 
mRNA-transfected cells were on average larger than the control group $\left(382 \pm 41\right.$ vs. $\left.145 \pm 23 \mathrm{~mm}^{2}, \mathrm{P}<0.05\right)$. These findings demonstrate that the transfected cells have greater tumorigenicity than the wild HeLa cells.

\section{Discussion}

Malignancy is a complex process of multi-factors and multi-stages. It involves widespread abnormal gene function. Understanding the molecular mechanisms leading to invasiveness and metastatic dissemination of carcinoma cells is important for the development of new therapeutic strategies against cancer.

In the present study, mRNA synthesized in vitro was transfected into HeLa cells to force expression of Nanog, and it was found that both mRNA and protein levels of Nanog were significantly increased in the Nanog mRNA-transfected HeLa cells. Then, we examined the effect of forced Nanog expression on the biological characteristics, such as colony formation capacity, cell migration and invasive ability, of HeLa cells. It was shown that the colony formation rate of HeLa cells with forced Nanog expression was higher than the wild control. There were more migrating and invasive transfected cells than in the wild controls. These results indicated that the forced expression of Nanog could increase the malignant behavior of HeLa cells.

CSCs play an important role in tumor progression $(3,15)$. To confirm whether the effect of forced Nanog expression on HeLa cell malignant behavior is associated with CSCs, we tested the tumor sphere forming ability of HeLa-Nanog cells. It was shown that the Nanog mRNA alone was able to increase the stemness (dedifferentiation) of cervical cancer cells, and the expression of endogenous Oct4, Sox2, and FoxD3 was significantly increased. This suggested that the pluripotency factor circuitry in the HeLa cells is at least partially active, allowing efficient dedifferentiation of HeLa cells by Nanog.

Studies have shown that the failure of chemotherapy in many malignant tumors was partially associated with abnormal expression of the MDR1 gene, which encodes the P-glycoprotein to pump anticancer agents out of the cells $(16,17)$. In the present study, we examined the expression of MDR1 in HeLa cells with or without Nanog mRNA transfection. We found that the expression of MDR1 was increased along with the improved expression of Nanog. This suggested that Nanog may be correlated with the expression of the MDR1 gene and further altered the chemosensitivity of human cervical cancer to cisplatin and paclitaxel. Although the underlying mechanism of Nanog in regulating MDR1 gene expression and chemoresistance remains unclear, these results indicate that aberrant expression of Nanog may be closely related to malignant characteristics, including multidrug resistance of cervical cancer, and inhibition of Nanog expression may be a new approach for sensitizing cervical cancer cells to chemotherapeutic drugs to reverse MDR in cervical cancer patients.

EMT plays a crucial role in promoting invasion and metastasis during tumor progression and is a process which tumor cells attenuate cell-cell adhesion to acquire a mesenchymal-like phenotype and disseminate into neighboring or distant tissues (18). Therefore, increased EMT disrupts E-cadherin-mediated cell-cell adhesion and converts epithe- lial-like cells into mesenchymal-like cells during tumor cell progression. In the present study, we found that the overexpression of Nanog resulted in increased EMT, with elevated $\mathrm{N}$-cadherin and vimentin and decreased E-cadherin in $\mathrm{HeLa}$ cells. In addition, the expression of Snail/Slug and Twist were also increased in Nanog overexpressing HeLa cells. It has been reported that Snail/Slug signaling regulates the EMT process (19). This evidence implies that Snail/Slug and Twist participate in regulating the EMT process to influence the metastasis of HeLa cells.

In conclusion, our present data suggested that the Nanog mRNA synthesized in vitro could activate the endogenous expression of Nanog and other transcription factors associated with pluripotency, followed by induction of the dedifferentiation of cervical cancer cells. This suggested that Nanog may be a positive regulator of cervical cancer cell dedifferentiation.

\section{Acknowledgements}

The present study was supported by the Hubei University of Medicine Juvenile Scientific and Technological Creativity team (2014 CXZ06), Educational Foundation of Hubei Province (B2015484) and Major Science and Technology Projects of infectious such as AIDS and viral hepatitis prevention and control, China (2013ZX10001-004-002-005).

\section{References}

1. Jemal A, Bray F, Center MM, Ferlay J, Ward E and Forman D: Global cancer statistics. CA Cancer J Clin 61: 69-90, 2011.

2. Hu Y and Fu L: Targeting cancer stem cells: A new therapy to cure cancer patients. Am J Cancer Res 2: 340-356, 2012.

3. Gilbert CA and Ross AH: Cancer stem cells: Cell culture, markers, and targets for new therapies. J Cell Biochem 108: 1031-1038, 2009.

4. Chambers I, Silva J, Colby D, Nichols J, Nijmeijer B, Robertson M, Vrana J, Jones K, Grotewold L and Smith A: Nanog safeguards pluripotency and mediates germline development. Nature 450: 1230-1234, 2007.

5. Zbinden M, Duquet A, Lorente-Trigos A, Ngwabyt SN, Borges I and Ruiz i Altaba A: NANOG regulates glioma stem cells and is essential in vivo acting in a cross-functional network with GLI1 and p53. EMBO J 29: 2659-2674, 2010.

6. Freberg CT, Dahl JA, Timoskainen S and Collas P: Epigenetic reprogramming of OCT4 and NANOG regulatory regions by embryonal carcinoma cell extract. Mol Biol Cell 18: 1543-1553, 2007.

7. Ben-Porath I, Thomson MW, Carey VJ, Ge R, Bell GW, Regev A and Weinberg RA: An embryonic stem cell-like gene expression signature in poorly differentiated aggressive human tumors. Nat Genet 40: 499-507, 2008.

8. Gu G, Yuan J, Wills M and Kasper S: Prostate cancer cells with stem cell characteristics reconstitute the original human tumor in vivo. Cancer Res 67: 4807-4815, 2007.

9. Jeter CR, Liu B, Liu X, Chen X, Liu C, Calhoun-Davis T, Repass J, Zaehres H, Shen JJ and Tang DG: NANOG promotes cancer stem cell characteristics and prostate cancer resistance to androgen deprivation. Oncogene 30: 3833-3845, 2011.

10. Ding Y, Yu AQ, Li CL, Fang J, Zeng Y and Li DS: TALEN-mediated Nanog disruption results in less invasiveness, more chemosensitivity and reversal of EMT in Hela cells. Oncotarget 5: 8393-8401, 2014.

11. Djurovic S, Iversen N, Jeansson S, Hoover F and Christensen G: Comparison of nonviral transfection and adeno-associated viral transduction on cardiomyocytes. Mol Biotechnol 28: 21-32, 2004.

12. Hoshijima M, Ikeda Y, Iwanaga Y, Minamisawa S, Date MO, $\mathrm{Gu}$ Y, Iwatate M, Li M, Wang L, Wilson JM, et al: Chronic suppression of heart-failure progression by a pseudophosphorylated mutant of phospholamban via in vivo cardiac rAAV gene delivery. Nat Med 8: 864-871, 2002. 
13. Zangi L, Lui KO, von Gise A, Ma Q, Ebina W, Ptaszek LM Später D, Xu H, Tabebordbar M, Gorbatov R, et al: Modified mRNA directs the fate of heart progenitor cells and induces vascular regeneration after myocardial infarction. Nat Biotechnol 31: 898-907, 2013.

14. Li B, Xu L, Lu WY, Xu W, Wang MH, Yang K, Dong J, Ding XY and Huang YH: A whole-mechanical method to establish human embryonic stem cell line HN4 from discarded embryos. Cytotechnology 62: 509-518, 2010.

15. Landry JJ, Pyl PT, Rausch T, Zichner T, Tekkedil MM, Stütz AM, Jauch A, Aiyar RS, Pau G, Delhomme N, et al: The genomic and transcriptomic landscape of a HeLa cell line. G3 (Bethesda) 3: 1213-1224, 2013

16. Pérez-Tomás R: Multidrug resistance: Retrospect and prospects in anti-cancer drug treatment. Curr Med Chem 13: 1859-1876, 2006.
17. Goda K, Bacsó Z and Szabó G: Multidrug resistance through the spectacle of P-glycoprotein. Curr Cancer Drug Targets 9: 281-297, 2009.

18. Acloque H, Adams MS, Fishwick K, Bronner-Fraser M and Nieto MA: Epithelial-mesenchymal transitions: The importance of changing cell state in development and disease. J Clin Invest 119: 1438-1449, 2009.

19. Casas E, Kim J, Bendesky A, Ohno-Machado L, Wolfe CJ and Yang J: Snail2 is an essential mediator of Twist1-induced epithelial mesenchymal transition and metastasis. Cancer Res 71: 245-254, 2011. 